\title{
OPTICAL PROPERTIES AND SPECTROSCOPY OF NANOMATERIALS
}


This page intentionally left blank 


\section{OPTICAL PROPERTIES AND SPECTROSCOPY OF NANOMATERIALS}

\section{Jin Zhong Zhang}

University of California, Santa Cruz, USA

\section{WF world Scientific}




\section{Published by}

World Scientific Publishing Co. Pte. Ltd.

5 Toh Tuck Link, Singapore 596224

USA office: 27 Warren Street, Suite 401-402, Hackensack, NJ 07601

UK office: 57 Shelton Street, Covent Garden, London WC2H 9HE

\section{British Library Cataloguing-in-Publication Data}

A catalogue record for this book is available from the British Library.

\section{OPTICAL PROPERTIES AND SPECTROSCOPY OF NANOMATERIALS}

Copyright (C) 2009 by World Scientific Publishing Co. Pte. Ltd.

All rights reserved. This book, or parts thereof, may not be reproduced in any form or by any means, electronic or mechanical, including photocopying, recording or any information storage and retrieval system now known or to be invented, without written permission from the Publisher.

For photocopying of material in this volume, please pay a copying fee through the Copyright Clearance Center, Inc., 222 Rosewood Drive, Danvers, MA 01923, USA. In this case permission to photocopy is not required from the publisher.

ISBN-13 978-981-283-664-9

ISBN-10 981-283-664-0

ISBN-13 978-981-283-665-6(pbk)

ISBN-10 981-283-665-9 (pbk)

Typeset by Stallion Press

Email: enquiries@stallionpress.com

Printed in Singapore. 
I wish to dedicate this book to all my teachers and mentors over the years, especially Mr. Wang Xue Yan, who first taught me how to read, count, and reason during the initial four years of my education. 
This page intentionally left blank 


\section{Preface}

This book is motivated by the need for an introductory level material focusing on the optical properties of nanomaterials and related spectroscopic techniques for upper level undergraduate and beginning graduate students who are interested in learning about the subject matter. While there are a number of excellent books on the market covering different aspects of nanomaterials, to date, there has not been a single monograph that specifically covers optical properties, optical spectroscopy and applications of nanomaterials.

Since optical properties are a major aspect of nanomaterials for both fundamental and technological reasons, I believe an introductory book specifically devoted to this subject is necessary and should be useful to both beginners and practitioners in the fields of nanoscience and nanotechnology. The objective is not to provide a comprehensive coverage or a review of all the nanomaterials studied to date, but rather to cover the very basics and illustrate the important fundamental principles and useful techniques with examples from recent literature. Given the fast pace of growth in nanoscience and nanotechnology, it is impossible to be comprehensive or inclusive. However, effort has been made to include as many significant and current examples as possible.

While nanomaterials are sometimes quite broadly defined to include inorganic, organic, biological, and various composite materials that involve a combination of these materials, this book will focus primarily on inorganic nanomaterials of semiconductor, metal and insulator. Some examples of more complex structures, including composites, will be briefly covered. 
This book can be used as a textbook or used by students on their own as long as they have some basic knowledge of quantum chemistry and optical spectroscopy. I have strived to provide a balanced coverage of both the basic principles as well as related experimental optical techniques so that students can gain knowledge and skills that are practical and directly useful to them in their learning and research. I have also made a special effort to ensure that this book is relatively easy to read and follow and, if used for teaching, can be taught in roughly one quarter or semester. I have used many figures and illustrations to help the readability. A fair number of references, again not meant to be complete or comprehensive, are given wherever appropriate.

I welcome feedback from readers and will attempt to incorporate them in future editions of this book, if such an opportunity arises.

Jin Zhong Zhang Santa Cruz, CA, USA December 2008 zhang@chemistry.ucsc.edu 


\section{Acknowledgments}

I would like to thank my mentors and many colleagues, collaborators, postdoctors, and students who have helped directly or indirectly with the writing of this book, through discussion, collaboration, and research work. An incomplete list of people to whom I wish to express my gratitude include: Ilan Benjamin, Rebecca Braslau, Mike Brelle, Frank Bridges, Guozhong Cao, Sue Carter, Ed Castner, Bin Chen, Jun Chen, Shaowei Chen, Wei Chen, Nerine Cherepy, Carley Corrado, Hai-Lung Dai, Elder De La Rosa, Hongmei Deng, Mostafa El-Sayed, Daniel Gamelin, Sarah Gerhardt, Daniel Gerion, Chris Grant, Claire Gu, Charles B. Harris, Greg Hartland, Eric J. Heller, Jennifer Hensel, Jianhua Hu, Thomas Huser, Dan Imre, Bo Jiang, Prashant Kamat, Alex Katz, Tav Kuykendall, Dongling Ma, Shuit-Tong Lee, Steve Leone, Chun Li, Can Li, Jinghong Li, Yadong Li, Yat Li, Tim Lian, Gang-yu Liu, Jun Liu, Xiaogang Liu, Tzarara Lopez-Luke, Glenn Millhauser, Rebecca Newhouse, Thaddeus Norman, Jr., Tammy Olson, Sergei Ostapenko, Umapada Pal, Cathy Phelan, Trevor Roberti, Lewis Rothberg, Nadya Rozanova, Archita Sengupta, George Schatz, Holger Schmidt, Adam Schwartzberg, Leo Seballos, Archita Sengupta, Chao Shi, Greg Smestad, Brian Smith, Jia Sun, Rebecca Sutphen, Chad Talley, Tony van Buuren, Changchun Wang, Zhong Lin Wang, Abe Wolcott, Fanxin Wu, Younan Xia, Xueming Yang, Kui Yu, Shuhong Yu, Yi Zhang, Zhongping Zhang, Yiping Zhao, Yingjie Zhu.

I wish to thank UCSC and a number of funding agencies for the partial financial support to do research in my lab over the years, and for the 
time I have spent on writing this book, including PRF-ACS, US NSF, US DOE and NSF of China.

I am grateful to my family, especially my parents, wife and daughters, for their unconditional support, love and understanding.

I wish to thank the book editor, Ms. Lakshmi Narayanan, for her wonderful and professional assistance. 


\section{Contents}

Preface vii

Acknowledgments $\quad$ ix

1. Introduction 1

2. Spectroscopic Techniques for Studying Optical Properties 11 of Nanomaterials

2.1. UV-visible electronic absorption spectroscopy 11

2.1.1. Operating principle: Beer's law 11

2.1.2. Instrument: UV-visible spectrometer 12

2.1.3. Spectrum and interpretation 14

2.2. Photoluminescence and electroluminescence 18 spectroscopy

2.2.1. Operating principle 18

2.2.2. Instrumentation: spectrofluorometer 18

2.2.3. Spectrum and interpretation 20

2.2.4. Electroluminescence (EL) 23

2.3. Infrared (IR) and Raman vibrational spectroscopy 24

2.3.1. IR spectroscopy $\quad 24$

2.3.2. Raman spectroscopy 26

2.4. Time-resolved optical spectroscopy 29

2.5. Nonlinear optical spectroscopy: harmonic generation 38 and up-conversion

2.6. Single nanoparticle and single molecule spectroscopy $\quad 40$

2.7. Dynamic light scattering (DLS) 41

2.8. Summary 42 
3. Other Experimental Techniques: Electron Microscopy and X-ray

3.1. Microscopy: AFM, STM, SEM and TEM

48

3.1.1. Scanning probe microscopy (SPM): $\quad 48$

AFM and STM

3.1.2. Electron microscopy: SEM and TEM 52

3.2. X-ray: XRD, XPS, and XAFS, SAXS 58

3.3. Electrochemistry and photoelectrochemistry 65

3.4. Nuclear magnetic resonance (NMR) and electron 67 spin resonance (ESR)

3.4.1. Nuclear magnetic resonance (NMR) 67

3.4.2. Electron spin resonance (ESR) 69

$\begin{array}{lll}\text { 3.5. Summary } & 72\end{array}$

4. Synthesis and Fabrication of Nanomaterials 77

4.1. Solution chemical methods 77

4.1.1. General principle for solution-based $\quad 77$ colloidal nanoparticle synthesis

4.1.2. Metal nanomaterials $\quad 79$

4.1.3. Semiconductor nanomaterials $\quad 84$

4.1.4. Metal oxides 90

4.1.5. Complex nanostructures 91

4.1.6. Composite and hetero-junction 95 nanomaterials

4.2. Gas or vapor-based methods of synthesis: CVD, 96

MOCVD and MBE

4.2.1. Metals 99

4.2.2. Semiconductors 99

4.2.3. Metal oxides 99

4.2.4. Complex and composite structures 101

4.3. Nanolithography techniques 101

4.4. Bioconjugation 102

4.5. Toxicity and green chemistry approaches 103

for synthesis

4.6. Summary 
5. Optical Properties of Semiconductor Nanomaterials

5.1. Some basic concepts about semiconductors

5.1.1. Crystal structure and phonons

5.1.2. Electronic energy bands and bandgap

5.1.3. Electron and hole effective masses

5.1.4. Density-of-states, Fermi energy, and carrier concentration

5.1.5. Charge carrier mobility and conductivity

5.1.6. Exciton, exciton binding energy, and exciton Bohr radius

5.1.7. Fundamental optical absorption due to electronic transitions

5.1.8. Trap states and large surface-to-volume ratio

5.2. Energy levels and density of states in reduced dimension systems

5.2.1. Energy levels

5.2.2. Density of states (DOS) in nanomaterials

5.2.3. Size dependence of absorption coefficient, oscillator strength, and exciton lifetime

5.3. Electronic structure and electronic properties

5.3.1. Electronic structure of nanomaterials

5.3.2. Electron-phonon interaction

5.4. Optical properties of semiconductor nanomaterials

5.4.1. Absorption: direct and indirect bandgap transitions

5.4.2. Emission: photoluminescence and Raman scattering

5.4.3. Emission: chemiluminescence and electroluminescence

5.4.4. Optical properties of assembled nanostructures: interaction between nanoparticles

5.4.5. Shape dependent optical properties

5.5. Doped semiconductors: absorption and 
5.6. Nonlinear optical properties 157

5.6.1. Absorption saturation and harmonic 157 generation

5.6.2. Luminescence up-conversion

5.7. Optical properties of single particles 160

5.8. Summary 165

6. Optical Properties of Metal Oxide Nanomaterials 181

6.1. Optical absorption 182

6.2. Optical emission 187

6.3. Other optical properties: doped and sensitized metal 194 oxides

6.4. Nonlinear optical properties: luminescence up-conversion (LUC)

6.5. Summary

7. Optical Properties of Metal Nanomaterials 205

7.1. Strong absorption and lack of photoemission 206

7.2. Surface plasmon resonance (SPR) 207

7.3. Correlation between structure and SPR: a theoretical 214 perspective

7.3.1. Effects of size and surface on SPR of metal 214 nanoparticles

7.3.2. The effect of shape on SPR

7.3.3. The effect of substrate on SPR 218

7.3.4. Effect of particle-particle interaction on SPR 218

7.4. Surface enhanced Raman scattering (SERS) 220

7.4.1. Background of SERS 220

7.4.2. Mechanism of SERS 221

7.4.3. Distance dependence of SERS 224

7.4.4. Location and orientation dependence 225 of SERS

7.4.5. Dependence of SERS on substrate 226

7.4.6. Single nanoparticle and single molecule SERS 229

7.5. Summary 
8. Optical Properties of Composite Nanostructures

8.1. Inorganic semiconductor-insulator and semiconductor-semiconductor

8.2. Inorganic metal-insulator

8.3. Inorganic semiconductor-metal

8.4. Inorganic-organic (polymer)

8.4.1. Nonconjugated polymers

8.4.2. Conjugated polymers

8.5. Inorganic-biological materials

8.6. Summary

9. Charge Carrier Dynamics in Nanomaterials

9.1. Experimental techniques for dynamics studies in nanomaterials

9.2. Electron and photon relaxation dynamics in metal nanomaterials

9.2.1. Electronic dephasing and spectral line shape 263

9.2.2. Electronic relaxation due to electron-phonon interaction

9.2.3. Photon relaxation dynamics

9.3. Charge carrier dynamics in semiconductor nanomaterials

9.3.1. Spectral line width and electronic dephasing

9.3.2. Intraband charge carrier energy relaxation

9.3.3. Charge carrier trapping

9.3.4. Interband electron-hole recombination or single excitonic delay

9.3.5. Charge carrier dynamics in doped semiconductor nanomaterials

9.3.6. Nonlinear charge carrier dynamics

9.4. Charge carrier dynamics in metal oxide and insulator nanomaterials

9.5. Photoinduced charge transfer dynamics

9.6. Summary 
10. Applications of Optical Properties of Nanomaterials 305

10.1. Chemical and biomedical detection, imaging 306 and therapy

10.1.1. Luminescence-based detection 306

10.1.2. Surface plasmon resonance (SPR) detection 309

10.1.3. SERS for detection 311

10.1.4. Chemical and biochemical imaging 315

10.1.5. Biomedical therapy 322

10.2. Energy conversion: PV and PEC 326

10.2.1. PV solar cells 326

10.2.2. Photoelectrochemical cells (PEC) 330

10.3. Environmental protection: photocatalytic and 331 photochemical reactions

10.4. Lasers, LEDs, and solid state lighting 335

10.4.1. Lasing and lasers 335

10.4.2. Light emitting diodes (LEDs) 336

10.4.3. Solid state lighting: ACPEL 339

10.4.4. Optical detectors 341

10.5. Optical filters: photonic bandgap materials 341 or photonic crystals

10.6. Summary

$\begin{array}{ll}\text { Index } & 359\end{array}$ 